\title{
A Propósito De Un Caso De Demencia \\ Frontotemporal - Variante conductual, Con Síntomas Amnésicos E Imágenes Cerebrales Tipo Alzheimer.
}

\author{
About a case of Frontotemporal Dementia - behavioral \\ variant, with amnesic symptoms and Alzheimer type \\ cerebral images: report of a case. \\ Nilton Custodio $^{1,2,}$ Rosa Montesinos ${ }^{2,3}$, Gustavo Hernández-Córdova ${ }^{2,4}$, Lizardo Cruzado ${ }^{5,6}$
}

\begin{abstract}
Frontotemporal dementia - behavioral variant (FTD-bv), is an important cause of disability in adults under 65 years of age. It is postulated as a neurological disease with predominantly psychiatric manifestations and, therefore, constitutes a frequent differential diagnosis in psychiatric pathologies of not early onset. Although traditionally the absence of memory impairment was considered among its diagnostic criteria - as opposed to Alzheimer's disease (AD) -, several studies have found until 15\% of prevalence of cases with frank mnemonic deterioration, as well as neuroimaging of one and the other can have overlap. Currently, the presence of "phenocopy syndromes" - clinical pictures similar to FTD-bv but without cognitive impairment-and cases of $A D$ with disinhibition and behavioral symptoms, make the clinical diagnosis more complex and suggest the insufficiency of the established diagnostic criteria and the necessary adoption of paraclinical criteria. To this purpose, we present an illustrative case of FTD-bv with memory impairment and Alzheimer-like neuroimages, and we review the pertinent bibliography.
\end{abstract}

Key words: Frontotemporal Dementia; Alzheimer's Disease; Memory. Rev Chil Neuro-Psiquiat 2020; 58 (1): 74-81.

Aceptado: 19/03/2010

Recibido: $24 / 02 / 2019$

Los Autores declaran no tener ningún conflicto de interés.

Servicio de Neurología, Instituto Peruano de Neurociencias, Lima, Perú.

Unidad de diagnóstico de deterioro cognitivo y prevención de demencia, Instituto Peruano de Neurociencias, Lima, Perú.

Servicio de Rehabilitación, Instituto Peruano de Neurociencias, Lima, Perú.

Laboratorios de Investigación y Desarrollo. Universidad Cayetano Heredia, Lima, Perú.

Facultad de Medicina. Universidad Peruana Cayetano Heredia. Lima, Perú.

Instituto Nacional de Salud Mental "Honorio Delgado - Hideyo Noguchi". Lima, Perú 


\section{Introducción}

己 a demencia frontotemporal (DFT) neurodegenerativos de inicio temprano y gran heterogeneidad, tanto en su cuadro clínico como su etiología y hallazgos histopatológicos e imagenológicos ${ }^{(1)}$. Es la demencia con mayor componente genético y produce afectación de la conducta, las funciones ejecutivas y el lenguaje. La DFT es la segunda causa de demencia después de la enfermedad de Alzheimer (EA) en menores de 65 años, por lo que tiene un importante impacto en personas de edad productiva ${ }^{(2)}$. Aunque los primeros casos fueron reportados por Pick en 1892, solo recientemente se ha incrementado la investigación sobre la DFT (3).

La DFT se manifiesta clínicamente en dos grandes síndromes: DFT variante conductual (DFTvc), con alteraciones predominantes del comportamiento social y del control ejecutivo; y la denominada Afasia Progresiva Primaria, con los subtipos: afasia progresiva no fluente, demencia semántica y afasia logopénica, donde el lenguaje es más afectado. En la evolución, las manifestaciones de los subtipos pueden converger, debido a que la degeneración tisular afecta masivamente a los lóbulos frontales y temporales ${ }^{(4)}$. Usualmente la evolución desde los primeros síntomas hasta el deceso del paciente oscila entre 5 y 7 años (2).

Estudios recientes hallan hasta en $15 \%$ de casos de DFT-vc, déficits en la memoria episódica similar a los de la EA, incluso desde estadios tempranos ${ }^{(5)}$. Estos déficits no se explican por alteraciones en la codificación y recuperación -donde interviene el córtex prefrontal- sino en la consolidación y almacenamiento de la memoria. Asimismo, los estudios imagenológicos han hallado afectación de regiones hipocampales y temporales en estos casos de DFT-VC, similares a las que se aprecian en casos de EA ${ }^{(6)}$. Esto contradice el paradigma diagnóstico en que la memoria no sufría alteraciones con la DFTvc y obliga a profundizar las investigaciones que caractericen mejor este tipo de cuadros. Otro punto aún pendiente de aclaración es la naturaleza de las llamadas "fenocopias" de la DFTvc, pacientes que presentan el mismo cuadro clínico pero con una evolución muy lenta - sin deterioro en actividades de la vida diaria- y sin correlato de atrofia cortical.

A propósito del caso de una mujer de 68 años, quien en su evolución ha presentado cuadro clínico compatible con DFT-vc con marcadas alteraciones mnésicas, revisamos la bibliografía y tratamos de encuadrar el caso desde la perspectiva actual.

\section{Caso clínico}

Mujer de 68 años, viuda, madre de tres hijos, con 10 años de escolaridad. Hace siete años se notaron cambios en su actitud: de ser amable y comedida, empezó a tratar con brusquedad a los clientes de su tienda de abarrotes. Progresivamente se mostraba más irritable y ruda incluso con sus parientes: con tendencia a la desinhibición y falta de empatía. Luego de dos años observaron que empezó a tratar con vulgaridad a sus nietos: los acusaba de ser pestíferos y piojosos. Divulgaba esto a personas ajenas a la familia lo que generaba muchas discusiones. La llevaron a un neurólogo quien diagnosticó "demencia senil" e indicó donepecilo, pero sin mejoría. Mostraba conductas erráticas: dromomanía persistente y prodigalidad con desconocidos. Asimismo empezó a mostrar problemas cognitivos: preparaba los alimentos cometiendo errores como agregar sal en vez de azúcar u olvidaba apagar la hornilla. La destinaron a labores de limpieza y observaron que las repetía de manera estereotipada.

Hace tres años se evidenció descuido de su aliño: no se bañaba sino a mucha insistencia y soltaba flatos en público. 
Presentaba conducta recolectora patológica: su habitación estaba llena de objetos inservibles. Si se la confrontaba, lo negaba y reaccionaba con irritación. Pero en general tendía al mutismo, a la prolongada apatía y la inmovilidad.

Hace dos años su deterioro fue más evidente: ya no podía ejecutar ninguna tarea doméstica. Empezó a presentar movimientos estereotipados con la boca en forma de chupeteo, sacando e introduciendo la lengua, en otros momentos contaba en voz baja: "1..2...3..4" con las manos detrás de la espalda. Mostraba hiperoralidad: gran apetencia por golosinas y gaseosas, se irritaba si no se las proporcionaban y subió de peso. También demostraba impaciencia: agredía si no la complacían de inmediato, y lloraba sin motivo aparente. Distintos facultativos le han diagnosticado "demencia vascular" o "enfermedad de Pick". Recibió quetiapina en dosis crecientes hasta $400 \mathrm{mg}$, además memantina $10 \mathrm{mg}$ y haloperidol 5 gotas en caso de inquietud marcada, con lo que se redujeron parcialmente su irritabilidad e impulsividad. Se le han pedido exámenes de laboratorio (biometría sanguínea, perfil renal, hepático, tiroideo, urinograma) con hallazgos dentro de lo normal, excepto su nivel bajo de vitamina B12 (87 pg/mL).

Actualmente, la paciente no presenta discurso espontáneo y mantiene estereotipias motoras y verbales. Puede comer y vestirse sola, no usa pañal. Muestra menos irritabilidad y agresividad, aunque exige golosinas y se muestra pueril. En el examen mental la hallamos despierta, inatenta, conectada al entorno, entiende órdenes simples y complejas, con hipomimia facial, bradicinética. Está desorientada en tiempo pero orientada en espacio y persona. $\mathrm{Su}$ lenguaje es escueto, no espontáneo. Se muestra apática, no sonríe.

A veces interviene de modo inapropiado señalando: "mi hijo es fiscal de la corte suprema" -lo que es falso-. Al final de la entrevista pregunta repetidas veces a su hijo si ha pagado la consulta y exige marcharse. $\mathrm{Su}$ impaciencia dificulta la evaluación mas detenida. No tiene insight ni conciencia de enfermedad. Al examen neurológico no se hallaron anormalidades motoras ni sensitivas, aunque los reflejos osteotendinosos estaban incrementados de forma asimétrica. Babinski y Hoffman negativos bilateralmente. Reflejos primitivos: enganche digital y succión; no se encontró prensión forzada. Equilibrio y coordinación conservada. Nervios craneanos son normales. Puntajes: Mini Mental State Examination (MMSE): 20/30. Addenbrooke's Cognitive Examination (ACE): 72/100. INECO Frontal Screening (IFS): 10/30. Clinical Dementia Rating (CDR): 2 (estadio moderado). Evaluación neuropsicológica: Severo compromiso de las funciones ejecutivas $\mathrm{y}$ atencionales; así como de la cognición social con leve compromiso del lenguaje y funciones visuoconstructivas; también existe marcado compromiso de la memoria verbal. En las Figuras 1,2 y 3 puede apreciarse imágenes de resonancia magnética nuclear encefálica de la paciente.

\section{Discusión}

La DFTve representa entre el 50 a $60 \%$ de casos de la DFT y predomina en varones respecto a mujeres $(2 \text { a } 1)^{(2)}$. En el cuadro clínico, la pérdida de empatía con los demás se combina con la desinhibición, la apatía, el aplanamiento emocional y la indiferencia afectiva (1). Asimismo, es frecuente el desarrollo de movimientos estereotipados y compulsivos y conductas de hiperoralidad. Se observa falta de autocrítica e insight, así como disminución del lenguaje espontáneo y hasta mutismo (3,7). Existe un porcentaje en que además se presentan tardíamente alteraciones del movimiento como enfermedad de la neurona motora, parálisis supranuclear progresiva y síndrome córticobasal ${ }^{(8)}$. Muchas veces se confunde 
Figura 1. Resonancia magnética nuclear, corte encéfalo axial-FLAIR: Moderada a severa atrofia frontal bilateral e insular a predominio derecho. Además, se observa hiper-intensidades de sustancia blanca a nivel periventricular y a nivel de parte anterior del cuerpo calloso con dilatación de ventrículos laterales.

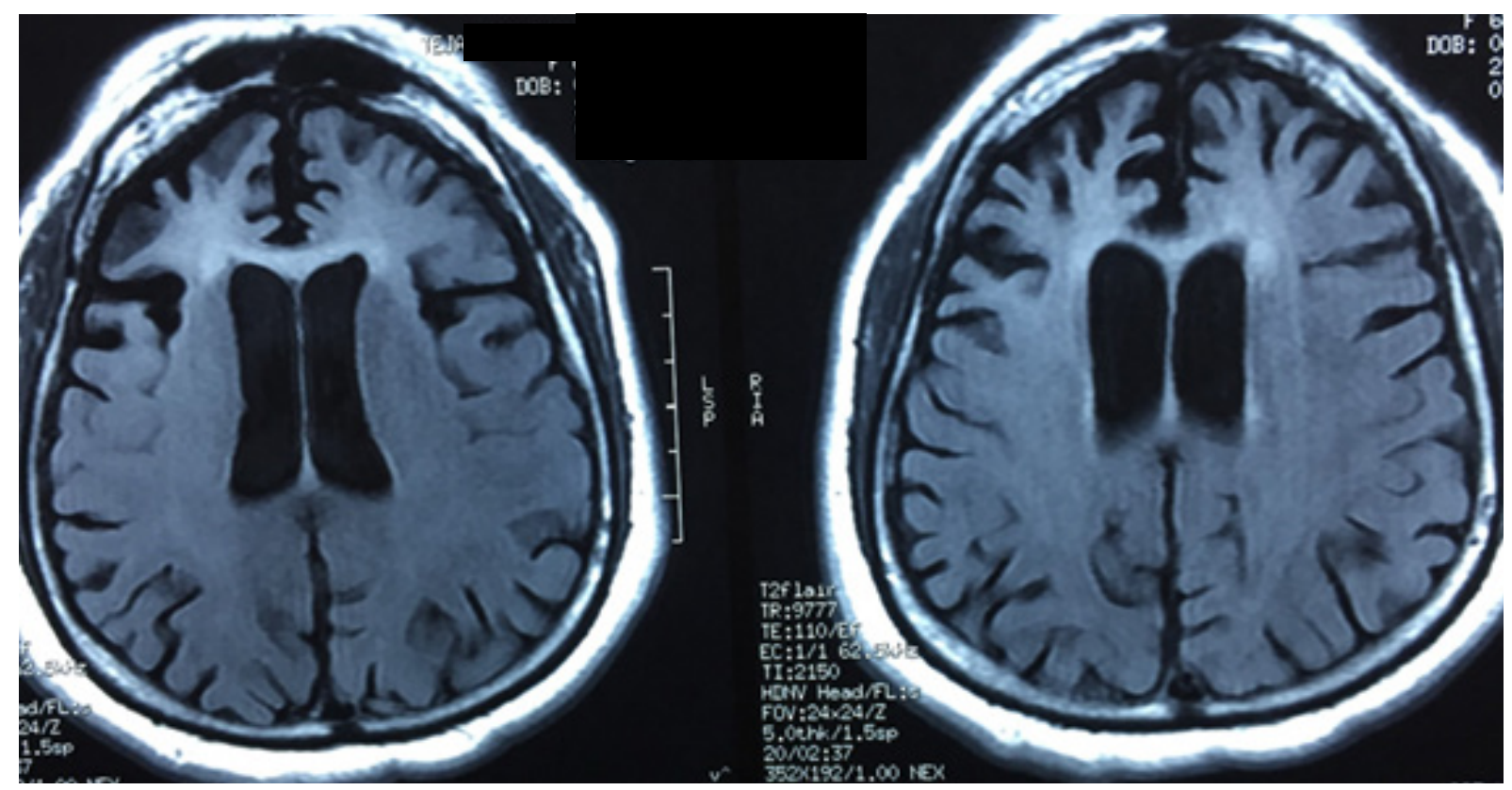

Figura 2. Resonancia magnética nuclear, corte encéfalo sagital-T1: Moderada a severa atrofia cortical frontal comparada con lóbulos posteriores.

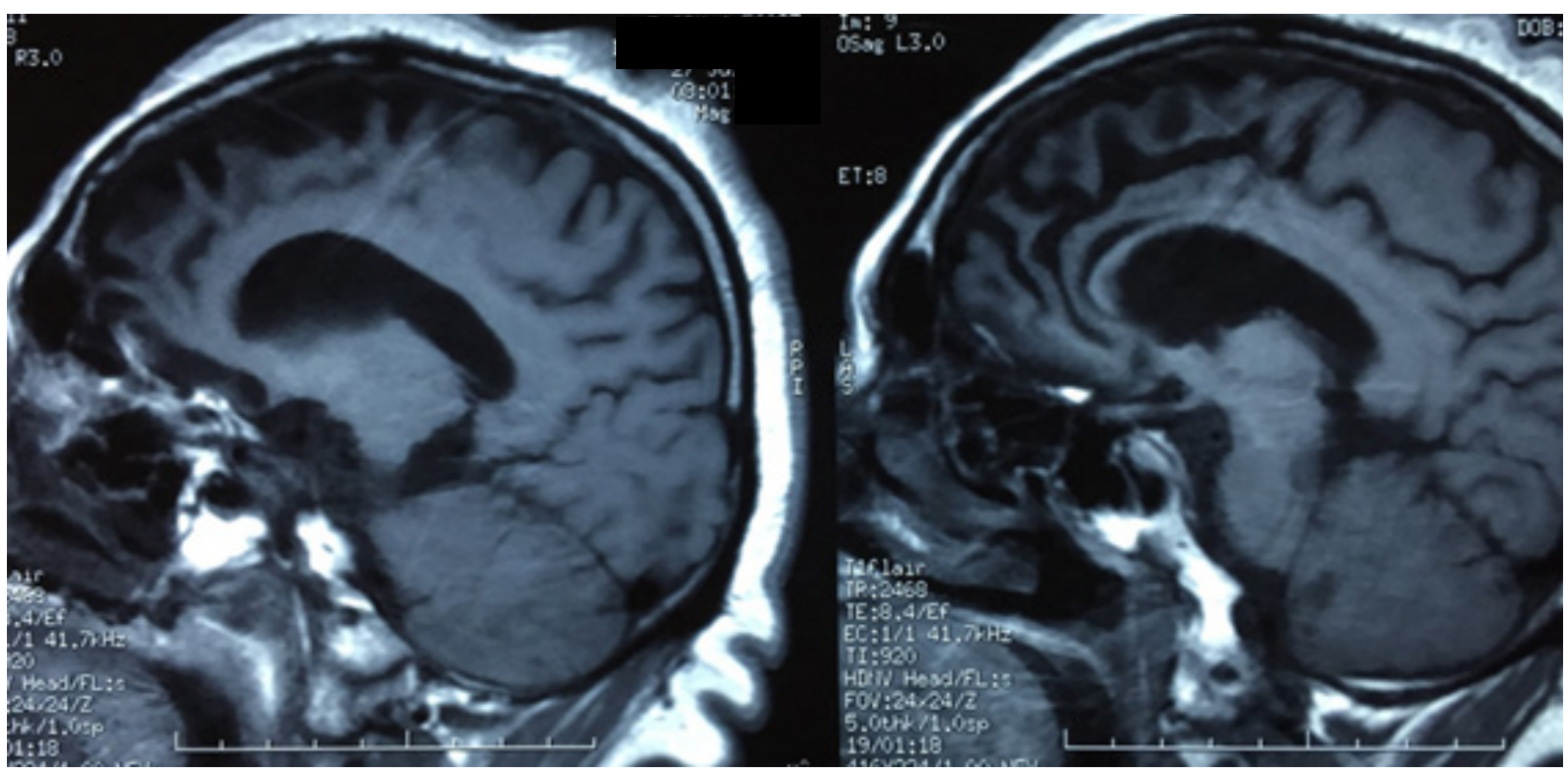


Figura 3. Resonancia magnética nuclear, corte encéfalo coronal-T2: Atrofia cortical frontal bilateral e insular a predominio derecho, con dilatación de ventrículos laterales y tercer ventrículo. Marcada atrofia de hipocampo.

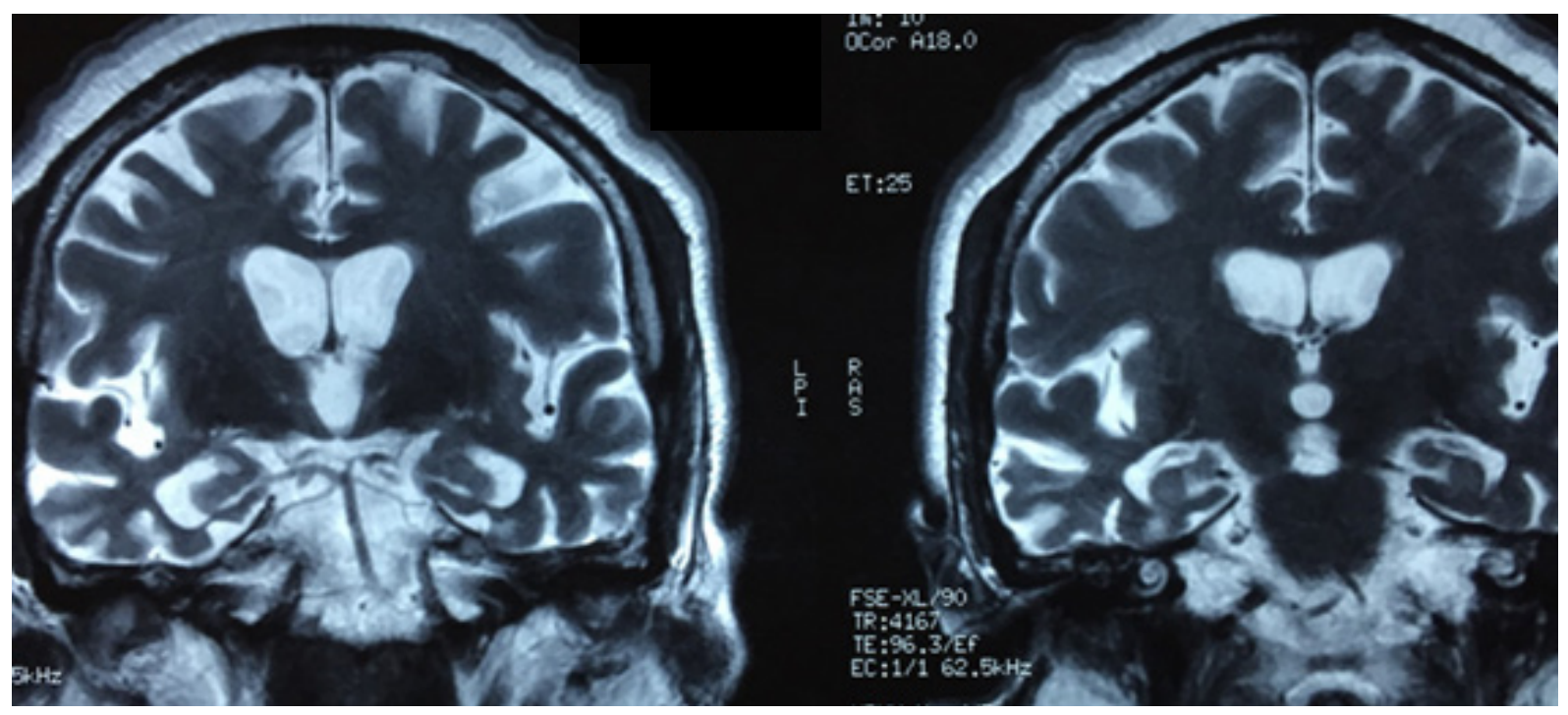

a la DFT con una serie de diagnósticos psiquiátricos (esquizofrenia de inicio tardío cuando aparecen síntomas psicóticos, manía por la desinhibición, depresión por la apatía, trastorno obsesivo compulsivo por las estereotipias), asimismo, la DFTvc en sus etapas tempranas puede originar cuadros secundarios de ludopatía, compras compulsivas, coleccionismo patológico, adicciones y otras patologías psiquiátricas $(9,10)$.

De acuerdos a los Criterios del Consenso Internacional (2011), para establecer un diagnóstico posible de DFTve se requiere al menos 3 de los siguientes criterios: desinhibición, apatía o abulia, pérdida de la actitud empática, conductas estereotipadas o compulsivas, hiperoralidad o cambios dietéticos, perfil neuropsicológico con alteración de funciones ejecutivas pero preservación de la memoria y habilidades visuoespaciales (7). En nuestro caso, se cumplen todos los criterios clínicos para un caso posible de DFT.

En cuanto a neuroimágenes, en la DFTvc han sido característicos los hallazgos de atrofia e hipometabolismo en los lóbulos frontales y polos anteriores de los lóbulos temporales, patrón no simétrico, sino con predominio del hemisferio derecho y de áreas como la ínsula, el giro cingulado, y zonas frontales específicas (1) se postula la susceptibilidad de estas zonas por la existencia de neuronas de von Economo, cruciales en la función del córtex prefrontal. En nuestro caso, las neuroimágenes con evidente atrofia frontotemporal e insular a predominio derecho certifican que se trata de un caso probable de DFT. La atrofia hipocampal, además, es evidente, hallazgo semejante al de $\mathrm{EA}^{(11)}$.

A nivel histopatológico se denomina Degeneración lobar frontotemporal (DFLT) a la afectación atrófica de estas estructuras, reflejo de disfunción de sistemas lisosomales $\mathrm{y}$ proteosomales con acumulación consiguiente de agregados neurotóxicos (12). El consenso neuropatológico actual establece tres grupos principales de acúmulos proteicos: de proteína Tau, de proteína fijadora del ADN (TP-43) y de la proteína fusionada en el sarcoma (FUS). El primer grupo corresponde al 35 a $50 \%$ de casos, el segundo es mayoritario, con más del $50 \%$ de 
casos y el tercer grupo aporta un porcentaje marginal ${ }^{(13)}$. A nivel genético también son tres los genes implicados: C9orf72, MAPT y GRN: si bien el 40\% de pacientes tienen historia familiar de demencia, solo en 10\% se halla dominancia autosómica clara. Los cromosomas mayormente implicados son el 3, 9 y $17^{(12)}$. Se han descrito diferentes patrones complejos de afectación tisular $\mathrm{y}$ acúmulos proteicos en función de los hallazgos genéticos descritos.

Como se ha señalado, es aún criterio para diferenciar DFTve de EA la presencia de una memoria episódica intacta. Sin embargo, 10 a $15 \%$ de casos confirmados patológicamente como DFT presentan alteraciones de memoria episódica en estadios iniciales de la enfermedad, esto ya se había notado incluso en los casos históricos de DFT ${ }^{(14)}$. Los resultados dispares en distintas series pueden deberse a la heterogeneidad de casos que conforman la DFT, variantes genotípicas y los diferentes modos de explorar las funciones mnésicas (6). En nuestro caso, la evaluación neuropsicológica corroboró la afectación mnésica prominente. Este fenotipo amnésico parece estar asociado a expansiones del gen C9orf72 y acúmulos de proteína TDP-43, sobre todo en el hipocampo. ${ }^{(15)}$

Los hallazgos recientes, respecto a las "fenocopias" sugieren que en realidad son variantes de la misma DFTve pues comparten anomalías estructurales corticales frontotemporales así como anomalías neuropatológicas ${ }^{(16,17)}$. Esto es, habría un espectro de continuidad entre las "fenocopias" y la DFTvc, asimismo, existen interesantes aportes desde la orilla de las investigaciones sobre enfermedad de Alzheimer con variantes conductuales - disejecutivas y conductuales, es decir, enfermedad de Alzheimer pero con síntomas más bien típicos de la DFTve ${ }^{(18)}$ : como si hubiera un muy amplio espectro cuyos extremos son la EA y la DFT típicas pero que abarcan cuadros intermedios donde podría ubicarse nuestro caso, con síntomas típicos de DFT pero con deterioro de memoria semejante a EA. Sería ideal en nuestro caso completar el estudio con los marcadores genéticos respectivos para caracterizar debidamente nuestro caso. En la actualidad, se ha propuesto que la más adecuada identificación de los cuadros demenciales sea en base a marcadores biológicos y no en base a cuadro clínico meramente: esto abre puertas a múltiples posibilidades diagnósticas y exige no solo un afiatado examen clínico sino los avances tecnológicos consiguientes ${ }^{(19)}$.

No existe en nuestro medio un adecuado conocimiento entre los profesionales médicos a cargo del diagnóstico las demencias, especialmente las DFT ${ }^{(20)}$. Se puede considerar a la DFTve como un trastorno neurológico con presentación psiquiátrica. Es imprescindible que se difunda el debido conocimiento de las DFT para su adecuado diagnóstico y tratamiento. Es un tema complejo y que requiere detección temprana para evitar diagnóstico y tratamientos inadecuados. Adicionalmente, como una prometedora perspectiva científica, dado el traslape de síntomas entre la DFTve y varias patologías psiquiátricas, se abre la perspectiva heurística de una hipotética base genética al menos en parte compartida ${ }^{(10)}$. 


\begin{abstract}
Resumen
La Demencia frontotemporal - variable conductual (DFT-vc), es una importante causa de discapacidad en adultos menores de 65 años. Se postula como una enfermedad neurológica con manifestaciones predominantemente psiquiátricas y, por tanto, constituye un frecuente diagnóstico diferencial ante patologías psiquiátricas de inicio no temprano. Aunque tradicionalmente se consideraba entre sus criterios diagnósticos a la ausencia de deterioro de la memoria -como en la Enfermedad de Alzheimer (EA)-, diversos estudios registran hasta un $15 \%$ de prevalencia de casos con franco deterioro mnésico, igualmente las neuroimágenes de uno y otro cuadro demencial pueden tener un traslape. Actualmente la presencia de "síndromes de fenocopia" -cuadros clínicos semejantes a DFT-ve pero sin deterioro cognitivo- y casos de EA con sintomas de desinhibición y conductuales, tornan más complejo el diagnóstico clínico y sugieren la insuficiencia de los criterios establecidos para el diagnóstico y la necesaria adopción de criterios paraclínicos. Con ese fin presentamos un caso ilustrativo de DFT-vc con deterioro de la memoria, y revisamos la bibliografia pertinente.
\end{abstract}

Palabras clave: Demencia Frontotemporal; Enfermedad de Alzheimer; Memoria.

\title{
Referencias Bibliográficas
}

1. Warren JD. Frontotemporal dementia. BMJ. 2013;347:f4827.

2. Waldö ML. The Frontotemporal Dementias. Psychiatr Clin N Am. 2015; 38(2):193-209.

3. Olney NT, Spina S, Miller BL. Frontotemporal Dementia. Neurol Clin. 2017; 35(2): 339-374.

4. Bang J, Spina S, Miller BL. Non-Alzheimer's dementia 1: Frontotemporal dementia. Lancet. 2015; 386(10004): 1672-1682.

5. Hornberger M, Piguet O. Episodic memory in frontotemporal dementia: a critical review. Brain. 2012: 135; 678-692.

6. Fernández-Matarrubia M, Matías-Guiu JA, Cabrera-Martín MN, Moreno-Ramos T, Valles-Salgado M, Carreras JL,et al. Episodic Memory Dysfunction in Behavioral Variant Frontotemporal Dementia: A Clinical And FDG-PET Study. J Alzheimers
Dis. 2017;57(4):1251-1264

7. Rascovsky K, Hodges JR, Knopman D, Mendez MF, Kramer JH, Neuhaus J, et al. Sensitivity of revised diagnostic criteria for the behavioural variant of frontotemporal dementia. Brain. 2011; 134: 2456-2477.

8. Bott NT, Radke A, Stephens ML, Kramer JH. Frontotemporal dementia: diagnosis, deficits and management. Neurodegener Dis Manag. 2014 ; 4(6): 439-454.

9. Pose M, Cetkovich M, Gleichgerrcht E, Ibáñez A, Torralva T, Manes F. The overlap of symptomatic dimensions between frontotemporal dementia and several psychiatric disorders that appear in late adulthood. Int Rev Psychiatry. 2013; 25(2): 159-167.

10. Galimberti D, Dell'Osso B, Altamura AC, Scarpini E. Psychiatric Symptoms in Frontotemporal Dementia: Epidemiology, Phenotypes, and Differential 
Diagnosis. Biol Psychiatry. 2015; 15; 78:684-692.

11. Finger EC. Frontotemporal Dementias. Continuum. 2016;22(2):464-489.

12. Karageorgiou E, Miller BL. Frontotemporal Lobar Degeneration: A Clinical Approach. Semin Neurol. 2014; 34:189-201.

13. Mann DMA, Snowden JS. Frontotemporal lobar degeneration: Pathogenesis, pathology and pathways to phenotype. Brain Pathol. 2017; 27: 723-736.

14. Bertoux M, de Souza LC, Corlier F, et al. Two distinct amnesic profiles in behavioral variant frontotemporal dementia. Biol Psychiatry. 2014; 75: 582-8.

15. Elahi FM, Miller BL. A clinicopathological approach to the diagnosis of dementia. Nat Rev Neurol. 2017; 13(8): 457-476.

16. Steketee RME, Meijboom R, Bron EE, Osse RJ, Koning I, Jiskoot LC, et al. Structural and functional brain abnormalities place phenocopy frontotemporal dementia (FTD) in the FTD spectrum. NeuroImage Clinical. 2016; 11: 595-605.
17. MeijboomR, Steketee RME, de Koning I, Osse RJ, Jiskoot LC, de Jong FJ, et al. Functional connectivity and microstructural white matter changes in phenocopy frontotemporal dementia. Eur Radiol.2017; 27:1352-1360.

18. Ossenkoppele R, Pijnenburg YAL, Perry DC, Cohn-Sheehy BI, Scheltens NME, Vogel JW. The behavioural/dysexecutive variant of Alzheimer's disease: clinical, neuroimaging and pathological features. Brain. 2015: 138; 2732-2749.

19. Custodio N. Los nuevos criterios para el diagnóstico de enfermedad de Alzheimer no dependen de los síntomas clínicos. Rev Neuropsiquiatr. 2018; 81: 55-57.

20. Custodio N, Becerra-Becerra Y, Cruzado L, Castro-Suárez S, Montesinos $\mathrm{R}$, Bardales Y, et al. Nivel de conocimientos sobre demencia frontotemporal en una muestra de médicos que evalúan regularmente a pacientes con demencia en Lima-Perú. Rev Chil Neuropsiquiat. 2018; 56: 77-88.

Correspondencia:

Lizardo Cruzado

Instituto Nacional de Salud Mental "Honorio

Delgado - Hideyo Noguchi”. Lima, Perú.

Jr. Eloy Espinoza 709. Urb. Palao. San

Martín de Porres, Lima 15102.

lizardo.cruzado.d@upch.pe

(051) 995733555 